\title{
Structures and Multipoint Correlators for Turbulent Advection: Predictions and Experiments
}

\author{
Laurent Mydlarski, ${ }^{1, *}$ Alain Pumir, ${ }^{2}$ Boris I. Shraiman, ${ }^{3}$ Eric D. Siggia, ${ }^{4}$ and Zellman Warhaft ${ }^{1}$ \\ ${ }^{1}$ Department of Mechanical Engineering, Cornell University, Ithaca, New York 14853 \\ ${ }^{2}$ I. N. L. N., 1361 route des Lucioles, F-06560, Valbonne, France \\ ${ }^{3}$ Bell Laboratories, Lucent Technologies, 700 Mountain Ave., Murray Hill, New Jersey 07974 \\ ${ }^{4}$ Center for Physics and Biology, Rockefeller University, New York, New York 10021
}

(Received 13 July 1998)

\begin{abstract}
We present theoretical predictions and wind tunnel measurements of the 3-point correlator of fluctuating temperature in preheated turbulent flow. The dependence of this passive scalar correlator on the point configuration is calculated on the basis of a model Hopf operator describing its time evolution but can be understood in terms of the underlying frontlike structure of the temperature field. Model predictions reproduce the main features observed in experiment. The geometric insight into the structure of the fluctuating field provided by multipoint correlators suggests new approaches to the study of turbulence itself. [S0031-9007(98)07639-X]
\end{abstract}

PACS numbers: 47.27.Ak, 02.50.Fz

Turbulent advection and mixing is a technically important problem in engineering (e.g., combustion) and in geophysical flows (e.g., dispersion of pollutants). The statistical properties of scalar fluctuations (e.g., temperature or the concentration of a pollutant) has many similarities with the statistics of the turbulent velocity field itself [1,2]. Much work [3] has been devoted to understanding the scaling properties of turbulent fields which deviate from the simple dimensional form predicted by the Kolmogorov theory [3] (K41). The observed anomalous scaling is caused by the relative excess of large fluctuations on small scales - the intermittency phenomenon-which is often interpreted in terms of "structures" present in the flow, such as vortex filaments [4] for the velocity field and the gradient sheets or "fronts" for the scalar field [5,6] [see Fig. 1(a)]. Yet, the connection between the scaling and the spatial structure of the flow has remained elusive.

One way towards establishing a connection between scaling and structure is to go beyond the traditional study of correlations at two measurement points to the investigation of multipoint correlation functions. The

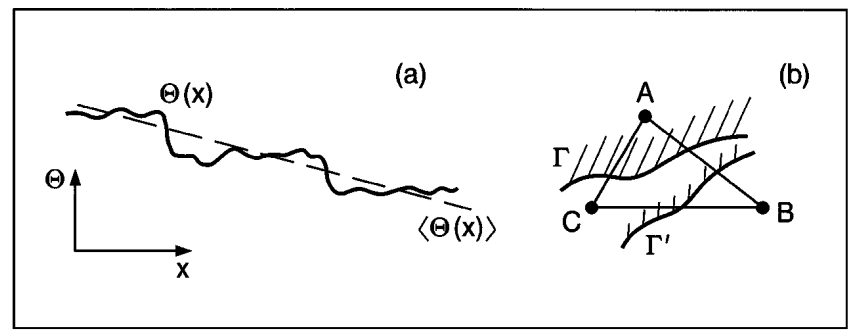

FIG. 1. (a) A cartoon of the instantaneous temperature profile $\Theta(x)$ exhibiting alternation of well-mixed plateau regions with the fronts where the gradient is concentrated. (b) The contributing of fronts to $\left\langle\theta\left(r_{A}\right) \theta\left(r_{B}\right) \theta\left(r_{C}\right)\right\rangle$ : If $\Gamma, \Gamma^{\prime}$ are fronts separating $\theta=1$ (hatched side) from $\theta=-1$ regions, front $\Gamma$ contributes 1 to the correlator, while $\Gamma^{\prime}$ contributes -1 . The net result depends on the front distribution and the geometry of the triangle. latter, in contrast with the conventional 2-point structure function, depend not only on the overall scale but also on the geometry of the point configuration [Fig. 1(b)] and thus contain more information. Moreover, the recent theoretical progress [7-10] on the passive scalar problem has led to the calculation of the multipoint correlators, making comparison between the experiment and theory both possible and necessary. The purpose of this Letter is to report and compare the experimental and theoretical results for the configuration dependence of the 3-point correlation function of the temperature field mixed by a nearly homogeneous and isotropic turbulent flow, in the presence of an imposed large scale temperature gradient.

The dynamics of the scalar field $\Theta$ advected by turbulent velocity $\vec{u}(\vec{r}, t)$ is governed by

$$
\partial_{t} \Theta+\vec{u} \cdot \nabla \Theta-\kappa \nabla^{2} \Theta=0,
$$

where $\kappa$ is the molecular diffusivity. The scalar field can be decomposed into the mean gradient and the fluctuating components: $\Theta(\vec{r})=\hat{G} \cdot \vec{r}+\theta(\vec{r})$ (where $\langle\theta\rangle=0$ ). The moments of the structure function for the scalar field, defined as $F_{n}(r)=\left\langle[\Theta(r)-\Theta(0)]^{n}\right\rangle$, have been studied experimentally [2,6,11]. $\quad F_{2}(r) \sim r^{2 / 3}$, in agreement $[1,11]$ with the K41 theory. However [11], $F_{3}(r) \sim r$, in contrast with the scaling exponent $5 / 3$ expected [12] from K41 theory. As a consequence, the skewness of the scalar derivative parallel to the mean gradient, $s_{\|} \equiv$ $\left\langle(\hat{G} \cdot \nabla \theta)^{3}\right\rangle /\left\langle(\hat{G} \cdot \nabla \theta)^{2}\right\rangle^{3 / 2}$ is of order 1 rather than decaying with Reynolds number as $\mathrm{Re}^{-1 / 2}$ predicted [12] by K41 theory. This effect implies that the anisotropy introduced by the large scale boundary conditions persists down to small scales which contradicts the very central concept of the Kolmogorov theory: the isotropy and universality of turbulence on small scales. This anomalous behavior, first observed in the atmosphere and then in the turbulent boundary layer over a heated plate [2,5], is associated with the transient formation of scalar gradient sheets aligned with the large scale gradient. The phenomenon is not an artifact of wall bounded shear flows since it 
is in no way diminished in the homogeneous turbulence produced in wind tunnels when supplemented by a large scale thermal gradient $[6,11]$ and, in fact, persists when the turbulent velocity is replaced by a synthetic Gaussian one in numerical simulations [13]. In the latter case, one has access to the entire temperature field which appears to be terraced with well mixed "plateaus" separated by the "cliffs" of scalar gradient [see Fig. 1(a)]. The simulations help legitimate theoretical approaches; one does not have to first understand the turbulent velocity before calculating its mixing properties [14-16]. For a Gaussian white in time velocity field, the general $n$-point correlation functions are governed by the Hopf equations which can be written down exactly $[15,17]$ and the exponents calculated as a function of that assumed for the velocity variance $[8,9]$. However, real turbulence is far from white, and to facilitate comparison with the experiment we shall rely on a class of phenomenological models $[10,18]$, which for the 3-point function lead to the Hopf equation in the following form:

$$
\left(L_{0}+\alpha L_{D}\right)\left\langle\theta\left(\vec{r}_{1}\right) \theta\left(\vec{r}_{2}\right) \theta\left(\vec{r}_{3}\right)\right\rangle=I_{3},
$$

where $L_{0}$ is the Batchelor-Kraichnan (BK) operator $[14,15]$ describing the random straining effect of the velocity field from the scales comparable to the point separations and $\alpha L_{D}$ models the incoherent effect of velocity fluctuations on smaller scales which act like eddy diffusivity. The right-hand side of Eq. (2) is the forcing term. Explicitly, the BK and eddy diffusivity operators are

$$
\begin{aligned}
L_{0} & =\sum_{i \neq j}^{N} D_{a b}^{(0)}\left(\vec{r}_{i}-\vec{r}_{j}\right) \partial_{r_{i}}^{a} \partial_{r_{j}}^{b}, \\
L_{D} & \equiv R^{2 / 3} \sum_{i<j}^{N} D_{a b}^{(2 / 3)}\left(\vec{r}_{i}-\vec{r}_{j}\right) \partial_{r_{i}}^{a} \partial_{r_{j}}^{b},
\end{aligned}
$$

where $D_{a b}^{(\epsilon)}(r)=D_{0}\left[(d+1-\epsilon) \delta_{a b}-(2-\epsilon)\left(r^{a} r^{b} /\right.\right.$ $\left.\left.|r|^{2}\right)\right]|r|^{2-\epsilon}$, and where $R^{2} \equiv \sum_{i<j}\left(\vec{r}_{i}-\vec{r}_{j}\right)^{2}$. This choice of $L_{D}$ corresponds to small scale velocity $\delta$ correlated in time and ensures that, in the limit where two points (say, $\vec{r}_{1}$ and $\vec{r}_{2}$ ) come close together, the correlation function behaves as $\left|\vec{r}_{1}-\vec{r}_{2}\right|^{2 / 3}$. For $\alpha \rightarrow \infty$ the Hopf equation (2) reduces, up to a multiplicative $R^{2 / 3}$ factor expressing the scaling of the correlation time, to the form it has in the Kraichnan's white in time velocity limit $i$ (with the K41 power spectrum) [15].

It was found that the homogeneous solution of Eq. (2), determined by the zero modes of the linear Hopf operator appearing on the left-hand side, dominates in the scaling range, making the inhomogeneous forced solution irrelevant [8-10]. Rotational invariance of the homogeneous problem plus the fact that the 3-point function is odd under parity imply that the latter must have angular momentum $l=1$. Using the translational symmetry, Eq. (2) can be reduced to an elliptic boundary value problem in two variables; after expressing the operators as finite differences, the resulting banded matrices are solved following Ref. [19]. The scaling index defined as $\left\langle\theta\left(a \vec{r}_{1}\right) \theta\left(a \vec{r}_{2}\right) \theta\left(a \vec{r}_{3}\right)\right\rangle=a^{\lambda}\left\langle\theta\left(\vec{r}_{1}\right) \theta\left(\vec{r}_{2}\right) \theta\left(\vec{r}_{3}\right)\right\rangle$ appears as a nonlinear eigenvalue in the boundary value problem. The exponent varies continuously from $\lambda=1$ for $\alpha=0$ to $\lambda \approx 1.38$ for $\alpha \rightarrow \infty$, as shown in Fig. 2 . The latter value of the exponent is too large, compared to the experimental value $(\lambda \approx 1)$, implying that the white in time velocity cannot accurately describe the experiment. The numerical solution for the $l=1$ zero mode also determines the configuration dependence of the correlator which will be compared with the experiment below.

In the wind tunnel with a mean flow along $\hat{x}$, and the transverse scalar gradient along $\hat{G} \equiv \hat{y}$; we placed two probes separated by a distance $y$, and recorded temperature fluctuations as a function of time. Using Taylor's hypothesis, one can thus measure the correlation between the three points: $A=\left(x_{1}+x_{2} / 2, y\right), B=\left(x_{2}, 0\right)$, and $C=(0,0)$. To remove the effects of slow drifts in the wind tunnel, the experimental data is best accumulated in terms of scalar differences between pairs of points chosen from among $(A, B, C)$. We define a generalized $3 \mathrm{D}$ order structure function by requiring that it satisfies full permutation symmetry. Introducing $\delta \theta_{1}=\theta_{B}-\theta_{C}$ and $\delta \theta_{2}=1 / \sqrt{3}\left(\theta_{B}+\theta_{C}-2 \theta_{A}\right)$, we construct $S_{3}=$ $\left\langle\left(\delta \theta_{1}+i \delta \theta_{2}\right)^{3}\right\rangle$ which has the desired symmetry. The real part $S_{3 R}=\left\langle\left(\theta_{A}-\theta_{B}\right)\left(\theta_{B}-\theta_{C}\right)\left(\theta_{C}-\theta_{A}\right)\right\rangle$ reduces ultimately to 2-point correlators, e.g., $\left\langle\theta(A) \theta(B)^{2}\right\rangle$. The imaginary part, however, is the irreducible 3-point correlator: $\left.S_{3 I}=\left\langle\delta \theta_{1}^{2} \delta \theta_{2}-\delta \theta_{2}^{3}\right)\right\rangle=C_{3}$ with $C_{3} \equiv$ $\langle\theta(A) \theta(B) \theta(C)\rangle$ provided that 1-point objects, such as $\left\langle\theta_{A}^{3}\right\rangle$, vanish as required by $\theta \rightarrow-\theta$ symmetry. At a fixed value of $y$, the correlation functions depend, assuming scaling, on $x_{1} / y$ and $x_{2} / y$. Thanks to the permutation and reflection symmetries, it suffices to present only one

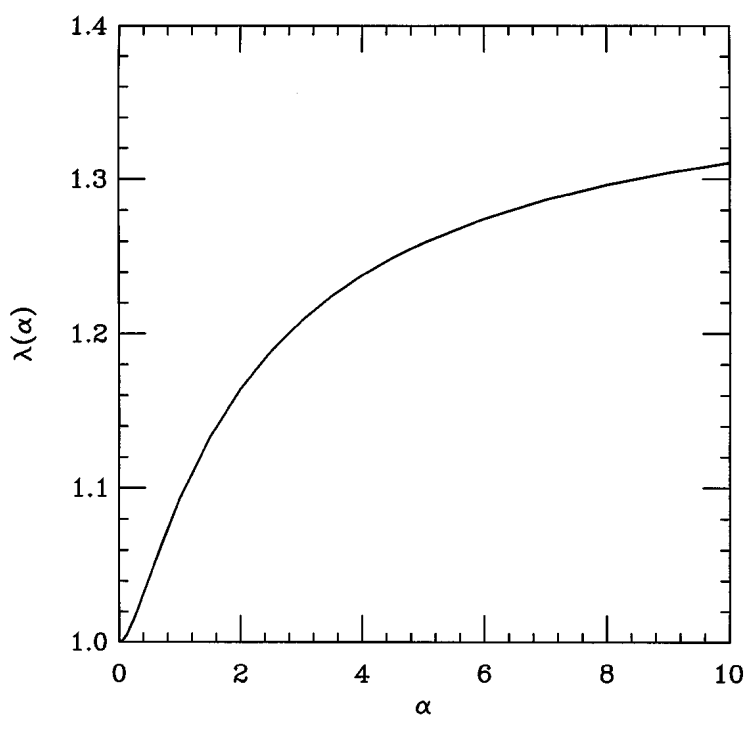

FIG. 2. The scaling exponent of the 3-point correlation function as a function of $\alpha$. The exponent tends to $\lambda \approx 1.38$ when $\alpha \rightarrow \infty$. Close to $\alpha=0, \lambda \approx 1+0.4 \alpha^{2}-0.35 \alpha^{5 / 2}$. 
quadrant $x_{1 / 2} / y \geq 0$. The data is nondimensionalized by dividing by $F_{3}(y)=\left\langle[\theta(0)-\theta(y)]^{3}\right\rangle / 6$ which is also used to measure the scaling exponent $\lambda \approx 1$. The theoretical function $C_{3}=\langle\theta(A) \theta(B) \theta(C)\rangle$ shown in Fig. 3 is to be compared with the experimentally measured $S_{3 I}$ shown in Fig. 4. The two have a similar qualitative behavior which includes the predicted and observed change of sign of the correlator from negative to positive with increasing $x_{1} / y$ as the observation points $B$ and $C$ separate, and the observation triangle evolves from acute to obtuse at vertex $A$. The best fit for $\alpha \approx 0.75$ (corresponding to $\lambda=0.27$ ) yields agreement on the level of $18 \%$.

It is instructive to compare the positions of the node $C_{3}=0$. Quite generally, one can prove that any function depending on configuration of three points and transforming as $l=1$ under rotations must vanish because of the additional permutation symmetry for an equilateral triangle [which corresponds to $\left(x_{1} / y, x_{2} / y\right)=(0,2 / \sqrt{3})$ ]. Indeed, the measured $S_{3 I}$ is zero (within experimental error) at that point. The measured correlator disagrees with the prediction in two ways: (a) It is nearly linear as a function of $x_{2} / y$ near the origin, compared to the sublinear $x_{2}^{2 / 3}$, behavior expected for $x_{2} \rightarrow 0$, as the $(B, C)$ pair of points merge together; (b) there is a considerable deviation for large $x_{1,2} / y$. These deviations are not unexpected because the scaling range is limited by the dissipation scale on the side of small $x_{2} / y$ and by the integral scale for $x_{1,2} / y$ large. Since the ratio of $y$ to the integral scale is $\approx 0.05$, one may expect a $20 \%$ level error for $\left(x_{1 / 2} / y\right)=5$ in the outer extremes of Figs. 3 and 4 , where $x_{1,2} / y \approx 5$. Additional measurements [11] at larger $y$ separations reveal an improved agreement for small $x_{2} / y$ at the expense of the $x_{1,2} / y \gg 1$ range, where the integral scale crossover effect becomes more severe, confirming our interpretation. Future experiments with more extensive scaling range should be closer to the theoretical limit and further theoretical work may compute subleading modes describing the crossover behavior.

Let us return to the physical interpretation of the configuration dependence of the 3-point correlator. The skewness of the temperature fluctuations is attributed to the transient appearance of sheets of intense scalar gradient (fronts) preferentially aligned with the normal to the large scale $\vec{G}$. Provided the instantaneous density of such sheets is low, their contribution to the 2-point skewness is $\left\langle[\Theta(r)-\Theta(0)]^{3}\right\rangle \sim r$, consistent with the observed scaling exponent. Our observation that the same scaling persists when all points are spread apart supports the notion that the sheets extend up to the integral scale. The simplest realization of the "front" picture assumes a single unit step in the $\Theta$ field appearing at an angle $\phi$ with respect to the large scale gradient with probability $1+a \cos \phi$ (corresponding to a pure $l=1$ mode). Let the front [see Fig. 1(b)] cut across the observation triangle with vertex $A$ on the positive side and $B, C$ on the negative: This gives

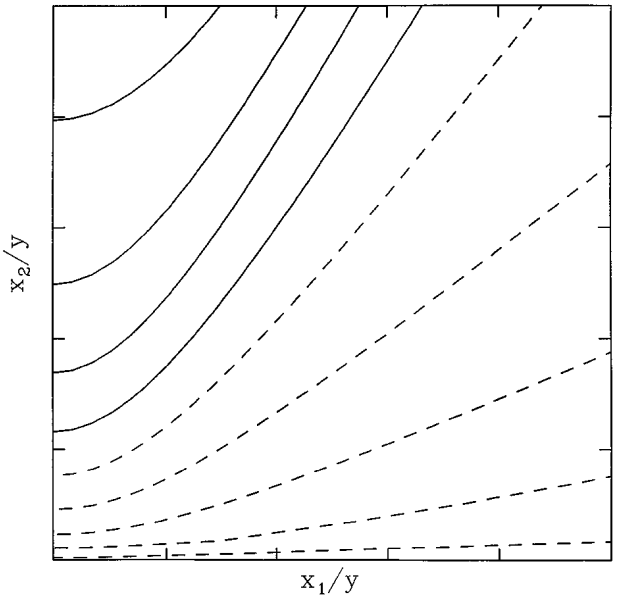

FIG. 3. Contour plot of the computed (for $\alpha=0.75$ ) correlation function $\langle\theta(A) \theta(B) \theta(C)\rangle$ for three coplanar points, $A=\left(x_{1}+x_{2} / 2, y\right), B=\left(x_{2}, 0\right)$, and $C=(0,0)$, as a function of $x_{2} / y$ (horizontal) and $x_{1} / y$ (vertical) in the range $0 \geq x_{1} / y, x_{2} / 4 \leq 5$. Integer values of the coordinates are indicated by ticks along the perimeter. Isocontours are separated by 0.2 . The scaling exponent is $\lambda=1.069$.

a positive contribution to $\langle\theta(A) \theta(B) \theta(C)\rangle$ and, if $B=C$, this would be the only possibility. However, as $B$ and $C$ separate there appears an increasing number of configurations where either $B$ or $C$ fall onto the positive side of the front, resulting in a negative contribution so that eventually $C_{3}$ changes sign. Although for each angle $\phi$ there exist two fronts with opposite signs, their probabilities are not equal and the net effect remains. The $C_{3}$ predicted by this naive front model is shown in Fig. 5 and fully captures the qualitative behavior of both the calculated and the measured correlators. Of course, the magnitude of the fronts has a nontrivial distribution of its own and the fronts are

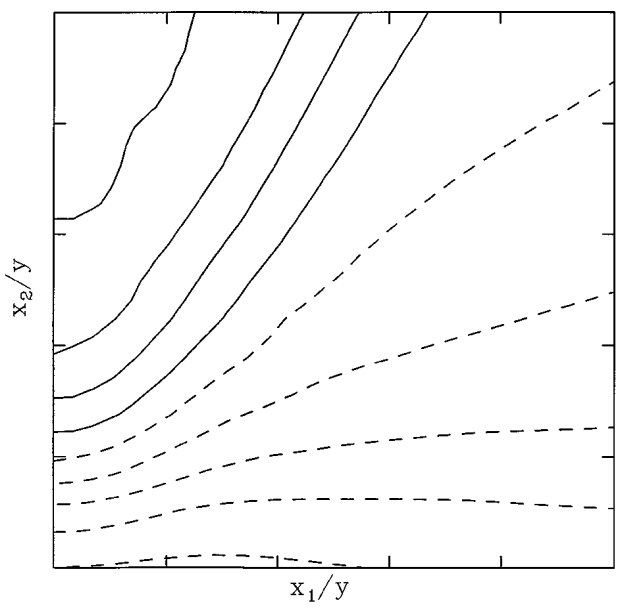

FIG. 4. Contour plot of the generalized 3-point structure functions $S_{3 I}$ measured in the experiment, plotted in the same range of $\left(x_{1} / y, x_{2} / y\right)$ as in Fig. 2. The isocontours are separated by 0.2 . The value of $y$ is $3.6 \mathrm{~mm}$; the integral length scale is $7.1 \mathrm{~cm}$, and the dissipative (Kolmogorov) scale is $\eta=0.2 \mathrm{~mm}$. 


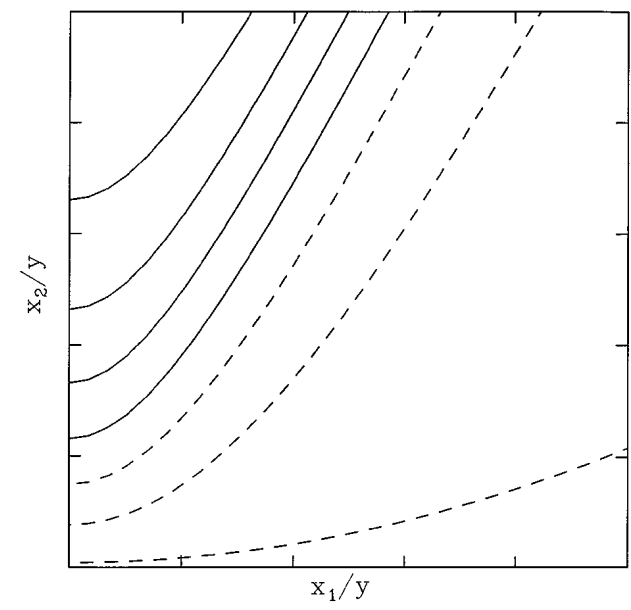

FIG. 5. Contour plot of the computed $C_{3}$ correlation function for the simple front model, shown in the same range of scales as in Fig. 2, with the same isocontour separation. This crude model captures well the main features visible in Figs. 3 and 4.

not just planes, yet the simple model with no adjustable parameters is remarkably predictive. Refining this simple model is not very useful, but ultimately the same information is implicit in the $N$-point correlators of the type considered in the present paper.

This Letter has introduced a new measure of small scale intermittency involving three points, which is sensitive to the structures - the gradient sheets-characteristic of turbulent mixing. Improved comparisons with experiment need not occur only by increasing the Reynolds number. Theory is able to furnish, to within an overall factor, the leading corrections through which the large scales contaminate the inertial ones, and so at the expense of a free parameter the fits should improve.

Our new correlation function can also be determined in a homogeneous shear flow where $\langle\vec{u}\rangle=G y \hat{x}$, where we imagine that the fluctuations in $\hat{x} \cdot \vec{u}$ play the role of temperature. Transport of $x$ momentum along $\hat{y}$ is analogous to the heat flux. Preliminary measurements [20] and simulations [21] of $\partial_{y} u$ also display a skewness of order 1, contrary to the postulate of inertial range isotropy, which has only been checked experimentally for $\langle\vec{u} \vec{u}\rangle$ [22]. It is therefore tempting to speculate that the vortical structures seen in turbulent flows are a consequence of the nonlinear evolutions of thin shear layers analogous to the gradient sheets of the scalar.
E.D.S. was supported by NSF DMR 9300711 and Z.W. by DOE. The numerical calculations were carried out on the Cray C98 thanks to a grant for computer time from IDRIS (France).

*Present address: Department of Mechanical Engineering, McGill University, Montreal, Canada.

[1] K. R. Sreenivasan, Proc. R. Soc. London A 434, 165 (1991).

[2] R. A. Antonia and C. W. van Atta, J. Fluid Mech. 84, 561-580 (1978).

[3] U. Frisch, Turbulence: The legacy of AN Kolmogorov (Cambridge University Press, Cambridge, England, 1995); M. Nelkin, Science 255, 566 (1992).

[4] E. D. Siggia, J. Fluid Mech. 107, 375 (1981); S. Douady, Y. Couder, and M.E. Brachet, Phys. Rev. Lett. 67, 983 (1991).

[5] P. G. Mestayer, J. Fluid Mech. 125, 475-503 (1982).

[6] C. Tong and Z. Warhaft, Phys. Fluids 6, 2165 (1994).

[7] R. H. Kraichnan, Phys. Rev. Lett. 72, 1016 (1994).

[8] K. Gawȩdzki and A. Kupiainen, Phys. Rev. Lett. 75, 3834 (1995).

[9] M. Chertkov et al., Phys. Rev. E 52, 4924 (1995).

[10] B. I. Shraiman and E. D. Siggia, C.R. Acad. Sci. 321, 279 (1995).

[11] L. Mydlarski and Z. Warhaft, J. Fluid Mech. 358, 135 (1998); Phys. Fluids (to be published).

[12] J. L. Lumley, Phys. Fluids 10, 855 (1967).

[13] M. Holzer and E. D. Siggia, Phys. Fluids 6, 1820 (1994); A. Pumir, Phys. Fluids 6, 2118 (1994).

[14] G. K. Batchelor, J. Fluid Mech. 5, 113 (1959).

[15] R. H. Kraichnan, Phys. Fluids 11, 945 (1968); J. Fluid Mech. 64, 737 (1974).

[16] R. H. Kraichnan, V. Yakhot, and S. Chen, Phys. Rev. Lett. 75, 240 (1995).

[17] B. I. Shraiman and E. D. Siggia, Phys. Rev. E 49, 2912 (1994).

[18] B. I. Shraiman and E. D. Siggia, Phys. Rev. Lett. 77, 2463 (1996); Phys. Rev. E 57, 2965 (1998).

[19] A. Pumir, Europhys. Lett. 37, 529 (1997); Phys. Rev. E 57, 2914 (1998).

[20] S. Tavoularis and S. Corrsin, J. Fluid Mech. 104, 349 (1981); S. Garg and Z. Warhaft, Phys. Fluids 10, 662 (1998).

[21] A. Pumir and B. I. Shraiman, Phys. Rev. Lett. 75, 3114 (1995).

[22] S. G. Saddoughi and S. V. Veeravalli, J. Fluid Mech. 268, 333 (1994). 